\title{
Editorial
}

\section{Neovascular AMD: Where do we go from here?}

\author{
Mark R Barakat ${ }^{1}$ and Pravin U Dugel ${ }^{2}$ \\ ${ }^{I}$ Mark R. Barakat, MD, practices with Retinal Consultants of Arizona in Phoenix. \\ ${ }^{2}$ Pravin U. Dugel, MD, is managing partner of Retinal Consultants of Arizona and a \\ founding member of Spectra Eye Institute in Sun City, AZ
}

In the age of anti-vascular endothelial growth factor (VEGF) therapy, we are fortunate as clinicians to have three great options in the treatment of neovascular age-related macular degeneration (AMD). Yet we still face a dilemma, in that we lack a truly sustainable treatment strategy. For various reasons, as a group we cannot maintain a monthly injection regimen for patients and are giving far less, approximately half of that, be it bevacizumab, ranibizumab, or aflibercept (Holekamp et al, 2014). Unfortunately, this comes at a cost: changing from monthly to as-needed injections has been associated with a decline in visual acuity almost back down to baseline (Singer et al, 2012; Silva et al, 2013).

There are three different approaches to addressing this problem we face in treating neovascular AMD. We can improve our results with anti-VEGF therapy by finding a better agent, overcome the treatment burden with improved delivery systems, or perhaps most importantly, broaden our current focus on VEGF to encompass other factors in the angiogenesis cascade as potential targets.

\section{Developing a better anti-VEGF}

The compound RTH258, formerly known as ESBA 1008, is a humanized single-chain antibody fragment inhibiting VEGF. In development by Alcon and Novartis, this firstof-its-kind agent binds with high affinity to all isoforms of VEGF-A and at $26 \mathrm{kDa}$ has a significantly smaller molecular size than current treatment options. In animal studies, more RTH258 than ranibizumab entered the retina, with a lower systemic exposure (Dugel, 2014) This may be due to the smaller size allowing better penetration into relevant target tissues.

In early trials of a single injection of RTH258 versus ranibizumab for neovascular AMD, the central subfield thickness on OCT was thinner and the visual acuity better with RTH258. Furthermore, eyes receiving the study drug went on average 30 days longer than ranibizumab-treated eyes before needing standard of care treatment, suggesting potentially longer duration. No safety imbalances were noted (Dugel, 2014). Similar results were seen when compared to aflibercept. A more definitive phase 3 trial is ongoing.

Another agent with small molecular size is Allergan's DARPin (Designed Ankyrin Repeat Proteins) product called AGN-150998 . In addition to its repeat structure, which allows it to be designed for a target duration, it also has a high affininty for VEGF, perhaps greater than the current medications available (data on file, Allergan). 
As earlier trials have been encouraging, a phase 3 trial (REACH) comparing AGN150998 to ranibizumab is currently ongoing (Allergan, https://clinicaltrials.gov/ ct2/show/NCT01397409).

Lastly, Conbercept is an anti-VEGF drug manufactured and already approved in China. A fusion protein of IgG with key domains of VEGF receptors, its design is similar to that of aflibercept. Phase 2 trials have shown potentially improved efficacy compared to bevacizumab (Kaiser et al, 2014).

\section{Improving therapeutic delivery}

Developing a better anti-VEGF delivery system is yet another way of addressing the overwhelming treatment burden seen with neovascular AMD. A novel mode of drug delivery introduces genetic material encoding the desired active compound into viruses, leveraging the virus' innate ability to enter and transfect target cells. The adenovirus, responsible for the common cold in its natural form, is particularly welladapted for this strategy of gene therapy treatment. Two companies, Genzyme and Avalanche, are both employing the adenovirus as a vector in this fashion, as either an intravitreal injection or an intraoperative subretinal injection.

Genzyme's approach employs an adenovirus containing the gene for sFLT01, a potent tyrosine kinase inhibitor that blocks the VEGF cascade further downstream than and hence could potentially be more effective than currently available anti-VEGF agents (Pechan et al, 2009). In animal studies of intravitreal injection of the vector, sFLT01 was expressed in the anterior chamber fluid as well as the macula, leading to decreased growth of laser-induced neovascular lesions (Lukason et al, 2011). Given the promising results, a phase 1 trial is underway with human subjects.

With a variation on the same concept, Avalanche is using a similar vector, but introducing it in the subretinal space, hoping to achieve a higher, localized transfection rate in the target area coupled with a lower incidence of side effects, such as inflammation. A small caliber needle is used to inject the virus under the retina after performing a core vitrectomy. After placement, the virus may last for more than a year and a half in animal studies, with no safety signals (Bennette et al, 1999; Lai et al, 2005). Reassuringly, no increase in geographic atrophy was noted with the long-term VEGF suppression (Bennette et al, 1999; Lai et al, 2005). In a phase 1 trial, the procedure yielded a reduction in the central retinal thickness comparable to that seen with the standard of care, while freeing the majority of patients from the need for rescue therapy for more than a year ( Chalberg et al, 2014). A phase 2 study has been planned.

Another delivery system consists of Neurotech's implantable protein factory, based on encapsulated cell technology (ECT) (Kauper et al, 2012). The implant, introduced with a small procedure, contains human retinal pigment epithelium (RPE)-derived, genetically modified cells, capable of producing any human protein. ECT consists of these cells within a semi-permeable tube that has pores strategically sized to allow free entry to the necessary oxygen and nutrients and exit of the produced therapeutic agent, while blocking larger immune components. Denying passage of immune factors that threaten to enter, attack and degrade the RPE-derived cells is a key function of ECT (Kauper et al, 2012). 
The protein factory in the ECT device has already demonstrated stable output more than 5 years after implantation in a prior geographic atrophy trial for a neurotrophic factor (NT-501) (Kauper et al, 2013). This was confirmed with viable RPE cells noted at the time of explantation (Kauper et al, 2013). While initial human trials with a single generation 2 implant that produces an anti-VEGF (NT-503 ECT) were encouraging, a subsequent double implant demonstrated even better VEGF inhibition, visual acuity and retinal thickness responses in neovascular AMD. On the heels of this observation, a generation 3 implant was designed to have a higher surface area to volume ratio, resulting in 6 times the delivery rate of the previous implant (Kauper et al, 2012). A phase 2 trial comparing the newest generation implant to aflibercept is in progress.

Yet another implantable device is the Replenish pump. It is implanted with a procedure much like with a Baerveldt glaucoma implant. Once in place, it is computer controlled, battery charged and can be replenished with an appropriate agent ( Flanigan, 2009). The Genentech Port is still another delivery system being developed that would allow refilling of a port placed under the conjunctiva (http://www.forsightlabs.com/pdfs/ FINAL\%20LAD\%20press\%20release\%201\%2013\%202012.pdf.).

\section{Expanding to other targets}

With a process as complex as the angiogenesis seen in neovascular AMD, it stands to reason that many factors other than VEGF are involved. There must be more than just VEGF inhibition required in treating this condition. To that end, a small potent anti-angiogenic molecule called squalamine, isolated from the dogfish shark (Squalus acanthias), is undergoing testing. It binds calmodulin intracellularly and prevents receptor activation, causing inhibition of a wide spectrum of factors, including VEGF and platelet derived growth factor (PDGF). Phase 2 trials of topical squalamine combined with ranibizumab versus ranibizumab alone showed improvements in vision and a decrease in retinal thickness on OCT (Slakter et al, 2015). A phase 3 trial is planned.

Squalamine is not the only agent directed at PDGF, as this growth factor plays a pivotal and previously unadressed part in the neovascular process. We now recognize that we upregulate PDGF with every anti-VEGF injection, which in turn recruits and matures pericytes. Pericytes cover the neovascular complex with a layer of protection that only leaves cells at the leading edge of the neovascular lesion exposed (Armulik et al, 2005). Only these unprotected cells, named tip cells, are sensitive to anti-VEGF therapy. The remaining majority of the endothelial cells, surrounded by a layer of pericyte armor, continues to mature even in the setting of treatment. In essence, antiVEGF monotheraphy only prunes the very edges of the neovascular complex, maturing the bulk of it.

Therefore, combination therapy with both an anti-VEGF and anti-PDGF agent seems an exciting prospect. The anti-PDGF treatment would strip the pericytes and sensitize the neovascular complex to the anti-VEGF agent, which in turn targets the underlying endothelial cells and causes neovascular regression. This has the potential of altering our entire approach to the management of AMD, marking a transition from an era of disease maintenance to disease modification. 
Out of several anti-PDGF compounds being tested by companies including Ophthotech, Santen, Regeneron, and Allergan, the one perhaps furthest along in development is Ophthotech's Fovista. Early trials showed potential, but most promising were the results from the phase $2 \mathrm{~b}$ trial presented in 2012 . With 449 subjects randomized to two doses of fovista in combination with ranibizumab versus ranibizumab alone, the trial is unique in size and design: as the largest phase $2 \mathrm{~b}$ trial in retina to date, it is also a superiority study, foregoing the non-inferiority trial design that one would commonly expect when comparing to a highly effective treatment. ${ }^{18}$ That said, at the highest fovista dose, mean change in vision with combination treatment proved superior to that seen with ranibizumab monotherapy, meeting the study's primary endpoint. There was a classic dose-reponse curve with fovista with a $62 \%$ improvement in visual acuity from baseline at the highest dosing, compared to ranibizumab alone. Pre-specified sub-group analyses of baseline lesions size and vision demonstrated that the benefit was seen across all group with no outliers. There were no local or systemic safety imbalances (Dugel, 2012).

For patients with greater than 4 lines and 5 lines of visual gain, there was a $71 \%$ and $190 \%$ relative benefit to combination therapy, respectively. Combination therapy also yielded a $119 \%$ relative benefit towards reaching $20 / 25$ visual acuity or better. Furthermore, fewer patients in the combination arm lost vision, highlighting a protective mechanism to the combination (Dugel, 2012).

Additional analysis of subretinal hyperreflective material (SHRM) on OCT, a biomarker thought to represent the neovascular lesion complex, showed that there was significantly more resolution with the addition of fovista. As for intraretinal, subretinal, and sub-RPE fluid on OCT, there was no difference between groups. As fovista is not an anti-permeability agent, this finding was expected.

Isolating the 4 and 5-line vision gainers, those in the ranibizumab monotherapy arm had OCT scans with a decrease in fluid but not in neovascular lesion size. Only small membranes, presumably not completely covered by pericytes, shrank with monotherapy. On the other hand, there was a significant reduction in neovascular membrane size as well as resolution of fluid in those gaining the same vision with combination treatment, This effect on lesion size has never before been described in clinical trials of any anti-VEGF product (Dugel, 2012).

For those patients that lost visual acuity, fibrosis and scar formation was the driving factor in eyes receiving ranibizumab alone, whereas little, if any, scarring was seen in the combination arm ((Dugel, 2012); Chakravarthy et al, 2014). As PDGF is a potent fibrotic agent, it stands to reason that its blockade decreases both fibrosis and subsequent scar formation, explaining this observed difference in treatment arms.

In short, the combined inhibition of VEGF and PDGF raises the possibility of actually halting the progression and even eliminating the neovascular complex, not just the associated exudation. If, as in wound-healing, PDGF plays a key role in the fibrosis and scarring seen late in neovascular AMD, then blocking PDGF in addition to VEGF may have yet another profound disease-modifying impact by reducing disciform scarring. 
In conclusion, while the treatment of neovascular AMD has advanced drastically with the advent of three highly effective anti-VEGF agents, these agents come with a treatment strategy that simply may not be practically sustainable. Robust data of our behavior as clinicians underscores this dilemma that we face. One strategy to address this problem, exemplified by DARPin and RTH258, is to develop a potentially better anti-VEGF agent. Another approach, taken by Genzyme, Avalanche, and Neurotech, is genetic engineering. Finally, combination therapy, particularly with an anti-PDGF such as Ophthotech's Fovista, has shown great promise as a potential solution.

As we await more definitive data on these new, disease-modifying therapeutic approaches, one thing seems certain: we are on the threshold of changing the way we manage neovascular AMD.

\section{References}

Armulik A, Abramsson A, Betsholtz C (2005). Endothelial/Pericyte Interactions Circulation Research; 97: 512-523

Bennett J, Maguire AM, Cideciyan AV, et al (1999). Stable transgene expression in rod photoreceptors after recombinant adeno-associated virus-mediated gene transfer to monkey retina. Proc Natl Acad Sci U S A; 96(17):9920-5.

Chakravarthy U and Jaffe GJ (2014). Dual Antagonism of Platelet Derived Growth Factor (Fovista $1.5 \mathrm{mg}$ ) and Vascular Endothelial Growth Factor (Lucentis $0.5 \mathrm{mg}$ ) Results in Reduced Subretinal Fibrosis and Neovascular Growth. Presented at the American Academy of Ophthalmology Annual Meeting; New Orleans, LA.

Chalberg TW (2014). Anti-VEGF gene therapy: early clinical results using the ocular biofactory in wet AMD. Paper presented at: Angiogenesis, Exudation, and Degeneration; Miami, FL.

Dugel P (2012). Anti-Platelet Derived Growth Factor: Where Do We Stand? Presented at Retina Subspecialty Day at the American Academy of Ophthalmology Annual Meeting; Chicago, IL.

Dugel P (2014). Results of ESBA 1008, a single-chain antibody fragment for the treatment of neovascular AMD. Presented at: American Society of Retina Specialists 2014 Annual Meeting; San Diego, CA.

Flanigan J (2009). Biotech tries to shrug off setbacks. The New York Times.

Genentech (2012). Genentech announces first milestone payment to device-maker ForSight VISION4, Inc. in development of sustained delivery Lucentis. Available at: http://www. forsightlabs.com/pdfs/FINAL\%20LAD\%20press\%20release\%201\%2013\%20 2012.pdf.

Holekamp NM, Liu Y, Yeh WS (2014). Clinical utilization of anti-VEGF agents and disease monitoring in neovascular age-related macular degeneration. Am J Ophthalmol; 157(4):825-833. e1. Epub 2013 Dec 31.

\section{https://clinicaltrials.gov/ct2/show/NCT01397409}

Kaiser PK (2014). Anti-VEGF therapy in wet AMD and conbercept development. Presented at: Angiogenesis, Exudation, and Degeneration 2014; Miami, FL. 
Kauper K, Elliott S, McGovern C, et al (2012). Long-term, sustained intraocular delivery of escalating doses of VEGF antagonist using encapsulated cell technology implant for the treatment of choroidal neovascular diseases. Presented at the Association for Research in Vision and Ophthalmology Annual Meeting; Fort Lauderdale, FL.

Kauper K, McGovern C, Stabila P (2013). Continuous Intraocular Drug Delivery over 5 1/2 Years: Ciliary Neurotrophic Factor (CNTF)Production by Encapsulated Cell Technology Implants Treating Patients with Retinitis Pigmentosa and Geographic Atrophy. Invest Ophthalmol Vis Sci; 54(15):3295.

Lai CM, Shen WY, Brankov M, et al (2005). Long-term evaluation of AAV-mediated sFlt-1 gene therapy for ocular neovascularization in mice and monkeys. Mol Ther; 12(4):659-68.

Lukason M, Dufresne E, Hillard R, et al (2011). Inhibition of choroidal neovascularization in a nonhuman primate model by intravitreal administration of an AAV2 vector expressing a novel anti-VEGF molecule. Mol Ther; 19: 260-265.

Pechan P, Rubin H, Lukason M, et al (2009). Novel anti-VEGF chimeric molecules delivered by AAV vectors for inhibition of retinal neovascularization. Gene Ther; 16: 10-16.

Singer MA, Awh CC, Sadda S, et al (2012). HORIZON: an open-label extension trial of ranibizumab for choroidal neovascularization secondary to age-related macular degeneration. Ophthalmology;119(6):1175-83. Epub 2012 Feb 4.

Silva R, Axer-Siegel R, Eldem B, et al (2013). SECURE Study Group. The SECURE study: long-term safety of ranibizumab $0.5 \mathrm{mg}$ in neovascular age-related macular degeneration. Ophthalmology;120(1):130-9.

Slakter JS, Ciulla TA, Elman MJ, et al (2015). Final Results from a Phase 2 Study of Squalamine Lactate Ophthalmic Solution $0.2 \%$ (OHR-102) in the Treatment of Neovascular Age-related Macular Degeneration (AMD). Invest Ophthalmol Vis Sci;56(7 ):4805. 